\title{
Shear instabilities in Taylor-Couette flow
}

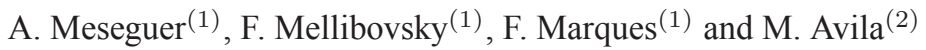 \\ (1) Dept. Applied Physics, Universitat Politecnica de Catalunya, Barcelona, Spain \\ (2) Max Planck Inst. for Dynamics and Self-Organization, Göttingen, Germany \\ alvar@fa.upc.edu
}

Summary. Subcritical instabilities in small gap Taylor-Couette (TCF) problem are studied numerically when both cylinders rotate in opposite directions. The computations are carried out for a radius ratio $\eta=r_{\mathrm{i}} / r_{\mathrm{o}}=0.883$. A first exploration is focused on the study of spiral flows originated from subcritical Hopf bifurcations of the basic circular Couette solution. The second exploration addresses the transition from laminar flow to the usually termed as spiral turbulence regime characterized by alternating laminar and turbulent spiral bands which coexist even in regions of the parameter space where the circular Couette flow is linearly stable.

\section{Formulation}

In TCF, an incompressible fluid of kinematic viscosity $\nu$ and density $\varrho$ is contained between two concentric rotating cylinders whose inner and outer radii and angular velocities are $r_{i}^{*}, r_{o}^{*}$ and $\Omega_{i}, \Omega_{o}$ respectively. The dimensionless parameters are the radius ratio $\eta=r_{i}^{*} / r_{o}^{*}$ and the inner and outer Reynolds numbers $\mathrm{R}_{\mathrm{i}}=d r_{i}^{*} \Omega_{i} / \nu$ and $\mathrm{R}_{\mathrm{O}}=d r_{0}^{*} \Omega_{o} / \nu$ of the cylinders. All variables are rendered dimensionless using the gap $d=r_{o}^{*}-r_{i}^{*}$ and viscous time $d^{2} / \nu$ as units for space and time, respectively. The dynamics of the flow is controlled by the incompressible Navier-Stokes equations

$$
\partial_{t} \mathbf{v}+(\mathbf{v} \cdot \nabla) \mathbf{v}=-\nabla p+\Delta \mathbf{v}, \quad \nabla \cdot \mathbf{v}=0 .
$$

In nondimensional cylindrical coordinates $(r, \theta, z)$, the basic circular Couette flow $(\mathrm{CCF})$ is $\mathbf{v}_{B}=\left(u_{B}, v_{B}, w_{B}\right)=(0, A r+B / r, 0)$, with $A=\left(\mathrm{R}_{\mathrm{o}}-\eta \mathrm{R}_{\mathrm{i}}\right) /(1+\eta)$ and $B=\eta\left(\mathrm{R}_{\mathrm{i}}-\eta \mathrm{R}_{\mathrm{o}}\right) /(1-\eta)\left(1-\eta^{2}\right)$. The flow is assumed to be $L^{*}$-periodic in the axial direction so that the dimensionless domain is $(r, \theta, z) \in \mathfrak{D}=\left[r_{i}, r_{o}\right] \times$ $[0,2 \pi) \times[0, \Lambda)$, where $\Lambda=L^{*} / d$ is the aspect ratio of the computational box. Arbitrary perturbations $\mathbf{u}$ of the base flow, $\mathbf{v}=\mathbf{v}_{B}+\mathbf{u}$, are expanded in a solenoidal spectral Fourier-Galerkin basis

$$
\mathbf{u}(r, \theta, z, t)=\sum_{l, n, m} a_{l n m}(t) \mathrm{e}^{\mathrm{i}\left(l k_{0} z+n \theta\right)} \mathbf{v}_{l n m}(r)
$$


for $(l, n, m) \in[-L, L] \times[-N, N] \times[0, M]$ and $k_{0}=2 \pi / \Lambda$. The time integration is carried out with a 4 th order linearly implicit time marching method [3]. Following former experimental works [1], the computations presented here were obtained for $\eta=0.883$, i.e., $r_{\mathrm{i}}=7.547$ and $r_{\mathrm{o}}=8.547$, with $\left(\mathrm{R}_{\mathrm{o}}, \mathrm{R}_{\mathrm{i}}\right) \in$ $[-3000,-1200] \times[0,1000]$ and $\Lambda=2 \pi / k_{0} \in[1.23,29.9]$. The spectral resolution used for the computation of seceondary laminar regimes lies within the intervals $\left(N_{r}, N_{\theta}, N_{z}\right) \in[28,48] \times[28,48] \times[16,32]$ radial $\times$ azimuthal $\times$ axial grid points, resulting in spectrally converged solutions. For the laminar-turbulent spiral patterns, a resolution within $\left(N_{r}, N_{\theta}, N_{z}\right) \in 20 \times[100,220] \times[100,220]$ was used, resulting in a dynamical system with up to $\mathcal{O}\left(10^{6}\right)$ degrees of freedom.

\section{Subcritical equilibria from modal instabilities of Couette flow}

The first exploration has consisted of a comprehensive numerical exploration of secondary finite amplitude solutions using Newton-Krylov methods embedded within arclength continuation schemes. Two different families of rotating waves have been identified: short axial wavelength subcritical spirals ascribed to centrifugal mechanisms and large axial scale supercritical spirals and ribbons associated with shear dynamics in the outer linearly stable radial region.

(a)

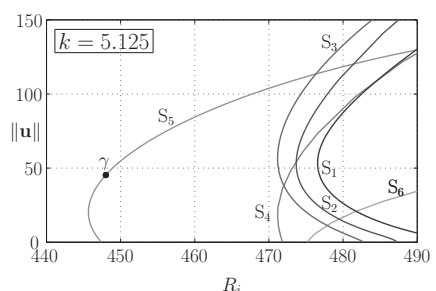

(b)

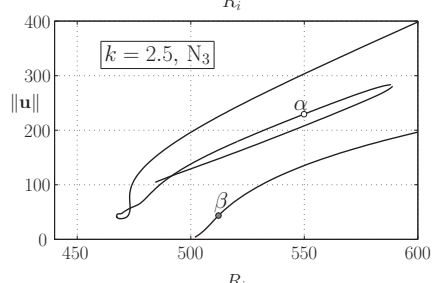

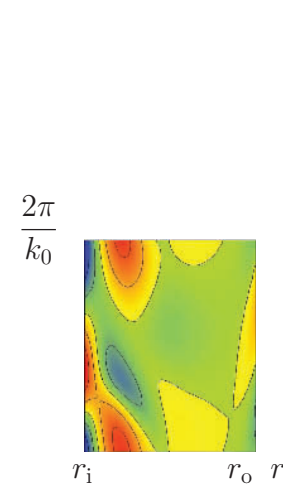

(c)

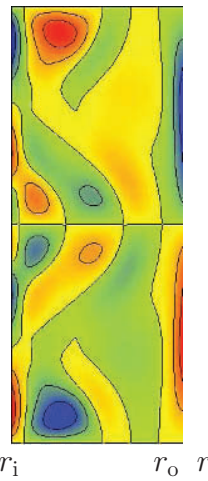

(d)

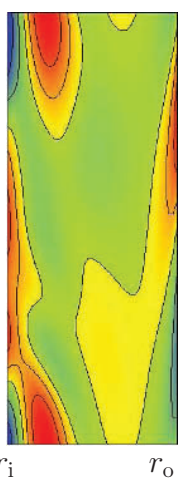

(e)

Fig. 1. (a) Norm of bifurcating spiral solutions of azimuthal wavenumbers $n \in[1,6]$ as a function of $\mathrm{R}_{\mathrm{i}}$ for $\mathrm{R}_{\mathrm{o}}=-1200$ and axial wavenumber $k=5.125$. (b) Same as (a) showing a disconnected ribbon solution branch $(\alpha)$ and a supercritical spiral branch $(\beta)$ for $k=2.5$. (c), (d) and (e) show azimuthal vorticity distributions on a $\theta=$ cnst. section of regimes $\gamma, \alpha$ and $\beta$ shown in (a) and (b), respectively.

As shown in Fig. 1a, all the spiral regimes originate from the circular Couette flow at Hopf bifurcations, some of them of subcritical nature. Their instability is clearly related to centrifugal mechanisms and their axial wavelength is consistent 
with the characteristic width of the inner centrifugally unstable region. Spiral flows with higher azimuthal and axial wave numbers tend to be less subcritical whereas spirals with lower azimuthal and axial wavenumbers, although not dominant at transition, clearly show more pronounced subcriticality. This motivated further exploration for lower values of $k_{0}$ in order to find the large-scale axial dynamics typical of spiral turbulence. However, straightforward continuation of the spirals for lower values of $k_{0}$ was unsuccessful, exhibiting turning points before $k$ reaches lower values. The computation of these new spiral shear modes required two different techniques, based on artificially cutting off high axial modes in the time evolution and quasistatic homotopy transformation in $\eta$, combined with an axial forcing. By including axial advection and reducing $\eta$ it was possible to find new spirals of larger wavelength. A very careful variation of these two combined effects sometimes succedeed in recovering low axial wavenumber rotating waves for our original problem. However, the described procedures have only been successful for $n_{0}=2$ and $n_{0}=3$ cases. Two families of new solutions have been found; one of them are spirals that bifurcate supercritically from the Couette flow. The other family consists of ribbons, apparently disconnected from the basic flow. Figure $1 \mathrm{~b}$ shows both families for $k_{0}=2.5$. The inner structures of the aforementioned equilibria are shown in Fig. 1c-f.

\section{Intermittent regimes from nonmodal instabilities}

The second exploration has consisted in triggering transition within the linearly stable region of the parameter space. This has been done for values of $R_{i}$ and $R_{O}$ for which experiments reported laminar-turbulent spiral coherence, also termed as spiral turbulence $[2,5,4]$. Numerical simulation with moderate resolution have managed to reproduce such a flow (see Fig. 2) within an axially periodic domain, thus confirming that this pattern is not necessarily induced by top or bottom lid effects. Figures $2 \mathrm{a}$ and $2 \mathrm{~b}$ show typical snapshots of computed SPT, conspicuously resembling the experimental results. The exploration reported here is summarized in Fig. 2c, where two parametric paths for $\mathrm{R}_{\mathrm{o}}=-3000$ and $\mathrm{R}_{\mathrm{o}}=-1200$ (labelled as $\Gamma_{1}$ and $\Gamma_{2}$, respectively) were followed. Both paths start within the shadowed region of Fig. 2c, where experiments [1] reported supercritical SPT regimes when increasing $R_{i}$ from below. Starting with a random perturbation at $\left(\mathrm{R}_{\mathrm{o}}, \mathrm{R}_{\mathrm{i}}\right)=(-3000,900)$ in $\Gamma_{1}$ and $\left(\mathrm{R}_{\mathrm{o}}, \mathrm{R}_{\mathrm{i}}\right)=(-1200,640)$ in $\Gamma_{2}$, the time integrations drove the flow towards SPT patterns in less than one viscous time unit. From those starting points, $R_{i}$ was quasistatically decreased and the time evolution of the flow was monitored for more than 6 viscous time units afterwards. Over $\Gamma_{2}$, SPT regimes followed exactly the same supercritical behaviour as the one observed in the experiments [1], where smooth decreasing of $\mathrm{R}_{\mathrm{i}}$ led to intermittency regimes (INT, characterized by localized turbulent spots), interpenetrating spirals (ISP) and relaminarization to the basic CCF profile. However, over $\Gamma_{1}$, the SPT flow was found to be sustained even below the linear stability boundary of the CCF, labelled as LSB in Fig. 2c. The $\mathrm{H}_{1}$ and $\mathrm{H}_{2}$ bulleted curves shown in Fig. 2c correspond to experimental hysteretic SPT boundaries when decreasing $R_{i}$ from above in [2] and [1], respectively. In particular, we found 


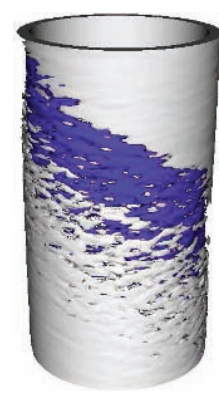

(a)

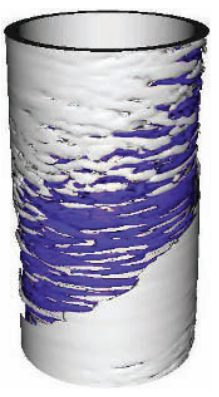

(b)

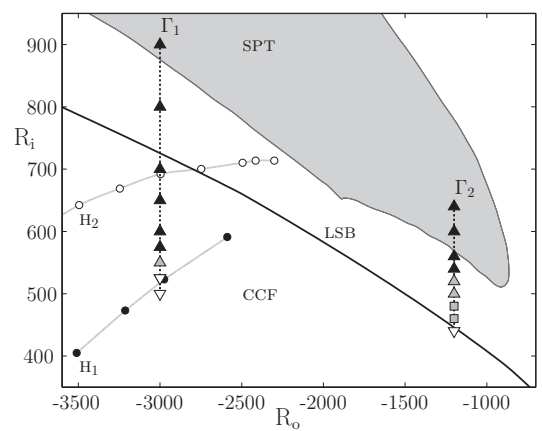

(c)

Fig. 2. Angular momentum $L=r v$ isosurfaces of typical SPT flows $(L>0$ and $L<0$ in white and blue, respectively). (a) Subcritical SPT at $\left(R_{\circ}, R_{i}\right)=(-3000,650)$ showing $L= \pm 1900$. (b) Supercritical SPT at $\left(\mathrm{R}_{\mathrm{o}}, \mathrm{R}_{\mathrm{i}}\right)=(-1200,600)$ showing $L= \pm 950$. (c) Explored regions in $\left(R_{\mathrm{o}}, \mathrm{R}_{\mathrm{i}}\right)$-parameter space. Black triangles, gray triangles, gray squares and white triangles correspond to SPT, INT, ISP and CCF flows, respectively.

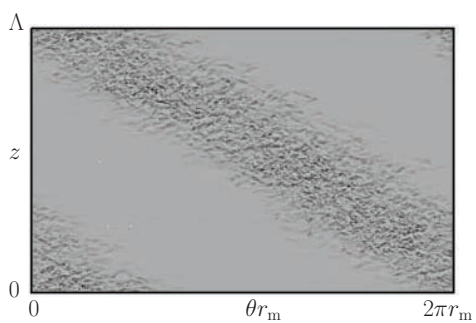

(a)

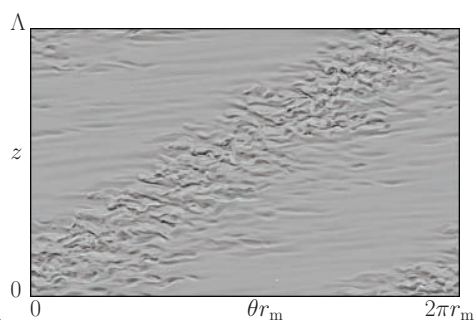

(b)

Fig. 3. Radial vorticity $(\nabla \times \mathbf{u})_{r}$ of SPT flows on the intermediate radial surface $r_{m}=8.21$. (a) $\left(\mathrm{R}_{\mathrm{o}}, \mathrm{R}_{\mathrm{i}}\right)=(-3000,650)$, with $(\nabla \times \mathbf{u})_{r} \in\left[-1.3 \times 10^{4}, 1.4 \times 10^{4}\right]$. and (b) $\left(\mathrm{R}_{\mathrm{o}}, \mathrm{R}_{\mathrm{i}}\right)=$ $(-1200,600)$, with $(\nabla \times \mathbf{u})_{r} \in\left[-6.6 \times 10^{3}, 6.1 \times 10^{3}\right]$. Dark and light regions represent negative and positive radial vorticity, respectively.

our computations to agree with the $\mathrm{H}_{1}$ boundary found by Coles. This work was supported by the Spanish Government grants FIS2007-61585 \& AP-2004-2235, and the Catalonian Government grant SGR-00024.

\section{References}

1. C. Andereck, et al., J. Fluid Mech., 164, 155 (1986).

2. D. Coles, J. Fluid Mech., 21, 385 (1965).

3. A. Meseguer, et al., Eur. Phys. J. Special Topics , 146249 (2007).

4. A. Prigent, et al., Phys. Rev. Lett., 89(1) 014501 (2002).

5. C. Van Atta, J. Fluid Mech., 25, 495 (1966). 